\title{
Myocardial perfusion 3-Tesla cardiac magnetic resonance vs. exercise electrocardiogram for diagnosics of coronary artery disease
}

\author{
Uwe Speiser, Achmed Abbas, Stefanie Jellinghaus, Burkhard Sievers, Ruth H Strasser, Steffen Schön \\ From 2011 SCMR/Euro CMR Joint Scientific Sessions \\ Nice, France. 3-6 February 2011
}

\section{Introduction}

Myocardial perfusion cardiac magnetic resonance imaging (MRI) is established as a high sensitive procedure to diagnose coronary artery disease (CAD). In practice exercise electrocardigram (XECG) is widely used as non invasive method to detect relevant coronary stenosis $(>50 \%)$.

The aim of this study was to investigate the diagnostic perfomance of adenosin stress 3-Tesla cardiac MRI for verification of ischaemia at coronary artery disease in comparison to XECG referred to coronary angiography as gold standard.

\section{Methods}

We included patients who received XECG, stress cardiac MRI and accomplished invasive coronary diagnostics. The MRI measurements at 3.0 T based on cine-modesequences in short axes images, rest and adenosin stress $(140 \mu \mathrm{g} / \mathrm{kg} \mathrm{bw} / \mathrm{min})$ perfusion and late gadolinium enhancement imaging (LGE, Magnevist $0,2 \mathrm{mmol} / \mathrm{kg}$ bw). Myocardial ischemia was defined as an area of perfusion deficit at stress MRI with negative late enhancement in areas of hypoperfusion (Panels A and B).

XECG was carried out on bicycle ergometer with a standardised protocol.

\section{Results}

40 patients ( $63 \pm 11$ years) were analysed prospectively, ten patients were assumed to have CAD, 30 had known $\mathrm{CAD}$ and were supposed to have a progress. A myocardial infarction in history was known at 14 patients,

XECG showed pathological findings in 24 cases $(60 \%)$, twelve of these had angina or severe dyspnea, eleven patients had significant horizontal or down sloping ST-depression, one patient had an unsustained ventricular tachycardia.

Coronary angiography investigated a significant CAD (stenosis $\geq 50 \%$ ) at 30 patients.

10 with normal XECG had relevant CAD. Sensitivity of XECG was $67 \%$, specifity $60 \%$. The positive predictive value (PPV) was calculated with $83 \%$, the negative predictive value (NPV) was $37 \%$.

Adenosin stress cardiac MRI revealed perfusion deficits in 32 patients (80\%). The sensitivity of MRI concerning coronary stenosis $\geq 50 \%$ was $93 \%$, the specifity $60 \%$. Two patients with inconspicuous adenosin stress cardiac MRI had significant CAD. The PPV of MRI was $88 \%$, the NPV $75 \%$. All 14 patients with prior myocardial infarction were detected by LGE-sequence (both sensitivity and specifity $100 \%$ ).

\section{Conclusion}

Myocardial perfusion magnetic resonance imaging at 3.0 Tesla is superior to exercise electrocardigram to diagnose relevant (stenosis $\geq 50 \%$ ) coronary artery disease. Cardiac MRI could also demarcate all patients with prior myocardial infarction by late enhancement.

Published: 2 February 2011

doi:10.1186/1532-429X-13-S1-P74

Cite this article as: Speiser et al:: Myocardial perfusion 3-Tesla cardiac magnetic resonance vs. exercise electrocardiogram for diagnosics of coronary artery disease. Journal of Cardiovascular Magnetic Resonance 2011 13(Suppl 1):P74

Dresden University of Technology, Dresden, Germany

(c) 2011 Speiser et al; licensee BioMed Central Ltd. This is an open access article distributed under the terms of the Creative Commons 